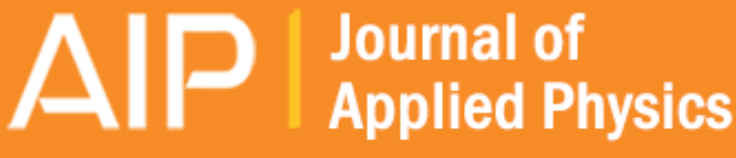

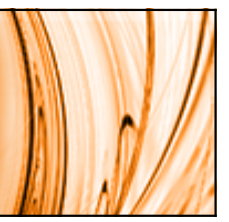

\section{Gaussian decomposition of beams and other functions}

David A. de Wolf

Citation: Journal of Applied Physics 65, 5166 (1989); doi: 10.1063/1.343144

View online: http://dx.doi.org/10.1063/1.343144

View Table of Contents: http://scitation.aip.org/content/aip/journal/jap/65/12?ver=pdfcov

Published by the AIP Publishing

\section{Articles you may be interested in}

A new form of Gaussian beam function as the base function for generic wave field analysis.

J. Acoust. Soc. Am. 127, 1845 (2010); 10.1121/1.3384335

Generating the Gaussian Basis Functions for Multi-Gaussian Beam Models

AIP Conf. Proc. 894, 878 (2007); 10.1063/1.2718061

Rigorous Gaussian-beam modeling of source functions for acoustic radiation and propagation

J. Acoust. Soc. Am. 82, S103 (1987); 10.1121/1.2024535

Transforming Gaussians into Wannier functions

J. Math. Phys. 19, 131 (1978); 10.1063/1.523530

Gaussian Functions in Molecular Integrals

J. Chem. Phys. 22, 148 (1954); 10.1063/1.1739831

\section{MIT LINCOLN}

LABORATORY CAREERS

Discover the satisfaction of innovation and service to the nation
- Space Control

- Air \& Missile Defense

- Communications Systems \& Cyber Security

- Intelligence, Surveillance and Reconnaissance Systems
- Advanced Electronics

- Tactical Systems

- Homeland Protection

- Air Traffic Control

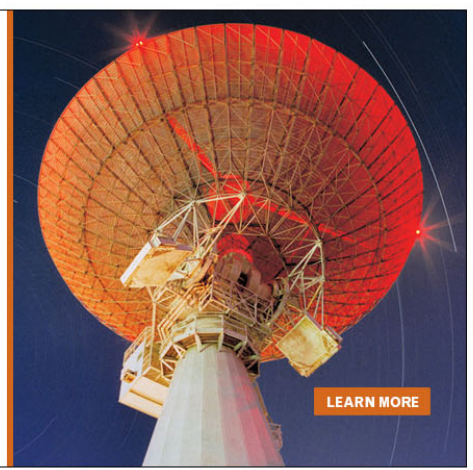




\title{
Gaussian decomposition of beams and other functions
}

\author{
David A. de Wolf \\ Bradley Department of Electrical Engineering, Virginia Polytechnic Institute and State University, \\ Blacksburg, Virginia $24061-0111$
}

(Received 10 November 1988; accepted for publication 27 February 1989)

The Gabor expansion of a function $f(x)$ decomposes it into a double sum over integers $m$ and $n$ of a product of basis functions $g(x-m X)$ and Fourier-series exponentials $\exp (2 \pi i n / X)$ for given spacing $X$. The choice of basis function determines the coeficients $a_{m n}$ of the expansion. If $f(x)$ is band limited, the couble sum can for all practical purposes be replaced by a single sum over Gaussian basis functions. This is extremely useful for expansion of multidimensional functions such as beams in phase space. Conditions of validity are given, and several examples illustrate the technique.

\section{MTRODUCTION}

This work concerns the expansion of a function into a set of Gaussian basic functions. Often, it is necessary or useful to expand a function into an orthonormal set of elementary basis functions. For example, Fourier analysis of a function uses trigonometric basis functions, and Laplace transform involves exponentials. In wave optics, the diffraction of a beam through an opening ${ }^{1}$ or through a phase screen ${ }^{2,3}$ is governed to good approximation by a Kirchhoff-Huygens convolution integral in which the beam amplitude is a factor. Expansion of this amplitude into the usual basis functions is often not useful, whereas a Gaussian shape factor yields a simple resuit, at least at very large distances. Another application concerns the propagation of a wave or particle beam in a refractive-index medium (for particles, the effective refractive index is determined by the electromagnetic potential). Phase-space techniques are used to calculate the progression of a phase-space density from its initial shape. This is relatively uncomplicated if the initial shape is Gaussian and the beam is narrow. ${ }^{4}$ Expansion of the beam into a set of narrow Gaussians is therefore very helpful. In a variety of dispersive, dissipative, and randomly refracting media, Fourier-transformike integrals occur with a kernel $\exp [-p(x)]$, where $p(x)$ is proportional to some (entire or fractional) power of $x$. In such cases, an expansion into Gaussians is useful, as it will result in a sum of analytically tractable integrals. Similar integrals are common to many branches of physics. Various other authors spanning a variety of fields have made use of expansions of beams into Gaussian beamlets. Popov and co-workers ${ }^{5-7}$ have put Gaussian beams through rays in a high-frequency approximation to the Helmholtz equation. Felsen, ${ }^{8,9}$ Mantica et al. ${ }^{10}$ and Pack and de Woll ${ }^{1}$ have exploited various forms of such expansions in recent work in optics. Various applications in geophysics have also made significant use of an expansion of bearlike functions into Gaussians. ${ }^{12-14}$ The dy. namics of quantum wave packets and associated calculations of molecular vibrational wave functions has been studied by expanding these wave packets into Gaussians in a series of publications by Heller, ${ }^{5}$ Davis and Heller, ${ }^{16-18}$ and associated authors. ${ }^{19-23}$ Applications to atomsurface scattering are discussed by Jackson and Metiu. ${ }^{24}$ Clearly, a widespread use is made of such expansions into
Gaussians, and we deem it useful to explore the conditions under which that is possible. The problem lies in the fact that Gaussian functions do not form an orthonormal basis for an arbitrary (but "well-behaved," e.g., continuous and differentiable) function $f(x)$. An approximate expansion can at best be given, and the conditions under which that is possible are the subject of this work.

\section{THE GABOR EXPANSION}

Recently, Bastiaans ${ }^{25}$ has brought the Gabor expan$\operatorname{sion}^{26}$ to the attention of workers in optics. Very briefly, Gabor proposed a bi-orthogonal expansion of a signal tunction $f(x)$,

$$
\begin{gathered}
f(x)=\sum_{m=-\infty}^{\infty} \sum_{n=-\infty}^{\infty} a_{m n} g(x-m X) \exp (i n Q x), \\
Q=2 \pi / X,
\end{gathered}
$$

where $g(x)$ is a chosen basis function (Gaussian in Gabor's paper), $X$ is a chosen spacing in variable $x$, and $Q$ is its wavenumber counterpart. Bastiaans ${ }^{25}$ and $J_{\text {anssen }}{ }^{27}$ have showed how to calculate the coefficients $a_{m n}$ for various choices of $g(x)$. The expression from which these are calculated follows from the definition

$$
\begin{aligned}
& h_{p}(x)=h(x+p X) \text { for }-X / 2<x<X / 2, \\
& h_{p}(x)=0 \text { orherwise }
\end{aligned}
$$

for any function $h(x)$, where $p$ is a (positive or negative) integer, and from the definition

$$
\tilde{h}(x, q)=\sum_{p=-\infty}^{\infty} f_{p}(x) e^{-i p q X} .
$$

It can then be shown that

$a_{m n}=\frac{1}{2 \pi} \int_{-x / 2}^{x / 2} d x \int_{\omega Q / 2}^{Q / 2} d q \frac{\tilde{f}(x, q)}{\tilde{g}(x, q)} e^{i m \phi X} e^{-i n Q x}$,

from which integral both Bastiaans ${ }^{25}$ and Janssen ${ }^{27}$ garnered rapidly converging expansions for these coefficients. Applications of (1)-(4) to optical tadiation through apertures are discussed by Einziger, Raz, and Shapira. ${ }^{28}$ Einziger and coworkers also discuss severai cases in which one of the summations in the expansion has only relatively few terms, e.g., as in the case of band-himited signals. Our purpose is to expand upon this and to indicate a special class of Gaussian 
basis functions with spacing and independent standard-deviation parameters that reduce the expansion to a single summation. Previous work ${ }^{25,28}$ appears only to have dealt with one-parameter Gaussians in the Gabor expansion.

\section{REDUCTION FOR BAND-LMITED FUNCTION}

The purpose of this section is to show that (1) can be reduced to a single summation

$$
f(x)=\sum_{m=-\infty}^{\infty} A_{m} g(x-m X)
$$

for a large class of functions $f(x)$, by appropriate choice of Gaussian functions $g(x)$, and that the coefficients $A_{m}$ are obtained from a sampling of $f(x)$ at discrete values spaced $X$ apart. The point of departure will be (4). Let us introduce the Fourier transform $H(q)$ of any function $h(x)$ as

$$
H(q)=\int_{-\infty}^{\infty} d x h(x) e^{-i q x}
$$

under the usual assumptions ${ }^{29}$ that the transform operation and its inverse are well defined. It is easily seen that the previously defined periodic functions (3) are related to the Fourier transforms (6) by

$$
\tilde{h}(x, q)=\frac{1}{X^{\prime}} \sum_{p=-\infty}^{\infty} H(q+p Q) e^{j(q+p Q) x} .
$$

From definitions (1) $-(4)$, it is also easily seen that

$$
\begin{aligned}
& f(x)=\sum_{m=-\infty}^{\infty} A_{m}(x) g(x-m X), \\
& A_{m}(x)=\frac{1}{Q} \int_{-Q / 2}^{Q / 2} d q \frac{\tilde{f}(x, q)}{\tilde{g}(x, q)} e^{i m q X} .
\end{aligned}
$$

If the (one-sided) bandwidth of $f(x)$, i.e., the extent of the Fourier transform in the spectral variable $q$, is limited below $q=Q_{B}$, and if $Q_{B}<\frac{1}{2} Q$, then $\bar{f}(x, q)$ in the integrand in $(8)$ can be replaced by $F(q)$, and the integral bounds may be replaced by $\pm Q_{E}$. This follows from substitution of the equivalents of $(7)$ for both $\tilde{f}(x, q)$ and $\tilde{g}(x, q)$ in the integrand in (8). Thus,

$$
\begin{aligned}
A_{m}(x)= & \frac{1}{Q} \int_{-Q_{n}}^{Q_{n}} d q \\
& \times\left(F(q) e^{i m q x} / \sum_{p=-\infty}^{\infty} \sigma(q+p Q) e^{i p Q x}\right) .
\end{aligned}
$$

At this stage, we choose a Gaussian basis function, namely, $g(x)=\exp \left(-x^{2} / 2 \sigma^{2}\right)$, with Fourier transform

$$
G(q)=(2 \pi)^{1 / 2} \sigma \exp \left(-\sigma^{2} q^{2} / 2\right) .
$$

Unlike Einziger and co-workers, ${ }^{28}$ we allow $\sigma$ to have an extra degree of freedom instead of defining it as $2 \pi / X$. We observe that

$$
\frac{G(q+Q)}{G(q)}=\exp \left[-\frac{1}{2} \sigma^{2}\left(Q^{2} \pm 2 q Q\right)\right]
$$

The factor

$$
\exp \left(-\frac{1}{2} \sigma^{2} Q^{2}\right)=\exp \left(-2 \pi^{2} \sigma^{2} / X^{2}\right),
$$

which is less than $2.7 \times 10^{-9}$ when $\sigma=X$ suggests that we restrict our choice of $\sigma$ to $\sigma \leqslant X$. The error in ignoring all but the $p=0$ term in the denominator of $(9)$ is then given by
(11) with $Q=Q_{B}$. Thus, the choice of $X$ is dictated by the requirement that $X<\pi / Q_{B}$, and the choice of $\sigma$ by $\sigma \leqslant X$. The error due to neglect of $p \neq 0$ terms in the denominator of (9) can be made vanishingly small by tailoring these two quantities for given $Q_{B}$. In practice, a compromise needs to be made between desired accuracy (small $X$, smaller $\sigma$ ) and affordable computer time $\left(X \cong \pi / Q_{B}, \sigma \approx X\right)$. If these criteria for $X$ and $\sigma$ are met, and the $p \neq 0$ terms are neglected, then we obtain

$$
A_{m *}=\frac{1}{Q} \int_{-\infty}^{\infty} d q \frac{F(q)}{G(q)} e^{i m q X}
$$

which is no longer a function of $x$. From this expression we can easily derive that

$$
A_{m}=(2 \pi)^{-1 / 2} \frac{X}{\sigma}\left[\exp \left(-\frac{1}{2} \sigma^{2} \frac{d^{2}}{d x^{2}}\right) f(x)\right]_{x=m X} .
$$

The formal exponent in (13) yields through fourth order

$$
\begin{aligned}
A_{m}= & (2 \pi)-1 / 2 \frac{X}{\sigma}\left(f(m X)-\frac{\sigma^{2}}{2} f^{\prime \prime}(m X)\right. \\
& \left.+\frac{\sigma^{4}}{8} f^{(4)}(m X)+\ldots\right),
\end{aligned}
$$

but in practice one may ignore the $O\left(\sigma^{2}\right)$, and higher terms as terms of similar magnitude have already been neglected in (12). Thus, the desired expansion (5) can be written as

$f(x) \approx(2 \pi)^{-1 / 2} \frac{X}{\sigma} \sum_{m}^{\infty} f(m X) e^{-(x-m X)^{2} / 2 \sigma^{2}}$

subject to the conditions $X<\pi / Q_{B}$ and $\sigma \leqslant X$, and the error terms are estimated by (11).

\section{THIN PHASE SCREEN}

A simple application of the above is to the propagation of a plane wave through a thin almost-transparent sereen containing turbulent air. The approach is well understood, ${ }^{2,3,30}$ and even fairly difficult parameters such as the intensity autocorrelation have been estimated under conditions of strong turbulence. ${ }^{31-3.3}$ Here, we simply apply the above to the calculation of the average intensity at large distances from this screen. For the one-dimensional case (turbulent cylinders in the $y$ direction ), the Kirchhoff-Huygens diffraction integral ${ }^{3}$ predicts

$$
\begin{aligned}
\langle I(\mathbf{r})\rangle= & \frac{k^{2}}{4 \pi^{2}} \int_{-\infty}^{\infty} d x_{1} \int_{-\infty}^{\infty} d x_{2} U\left(x_{1}, d\right) U^{*}\left(x_{2}, d\right) \\
& \times g_{0}\left(r-r_{1}\right) g_{0}^{*}\left(\mathbf{r}-r_{2}\right) \Gamma_{2}\left(\mathbf{r}, \mathbf{r}_{1}, r_{2}\right)
\end{aligned}
$$

where $U(x, d)$ is the aperture function at $z=d, g_{0}(R)$ is the free-space Green's function, and $\Gamma_{2}$ is the wave conerence function for reception at a distant point $r=(\boldsymbol{p}, L)$. If certain stibtleties such as infuence of inner scale of turbulence are ignored, then one may set ${ }^{34}$

$$
\Gamma_{2}=\exp \left(-\kappa C_{n}^{2} k^{2} d\left|x_{1}-x_{2}\right|^{5 / 3}\right),
$$

where $C_{n}^{2}$ is the atmospheric structure constant for turbulence and $k$ is the wave number ( $k$ is a constant of no importance here), and (16) becomes

$$
\langle I(x, L)\rangle=\frac{k x_{0}}{2 \pi L} \int_{0}^{\infty} d t \Gamma_{0}\left(\frac{D t}{2 \lambda_{T}}\right) e^{-\mid t t^{p / z}} e^{-i x t / \lambda_{T}},
$$


PHASE SCREEN

Mean angular spectrum

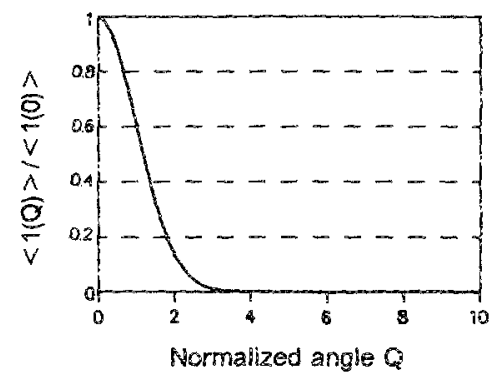

FIG. I. Normalized angular spectrum $\langle I(Q)\rangle /\langle I(0) /\rangle$ vs $Q=x / \lambda_{T}$ for thin phase screen. where $\Gamma_{0}$ is the coherence function for the aperture of diameter $D$, and $\lambda_{T}$ is a turbulence length parameter:

$$
\lambda_{T}=\left(L^{2 / k d}\right)^{1 / 2} \sigma_{T}^{6 / 5} \text {, with } \sigma_{T}^{2} \propto C_{n}^{2} k^{7 / 6} d^{11 / 6},
$$

a parameter describing the strength of the turbulence. When propagation distance $Z$ is very much larger than $D$, it is permissible to set $\Gamma_{0}=1$ and the integral becomes

$$
\langle I(x, L)\rangle=\kappa \int_{0}^{\infty} d t e^{-t^{5 / 3}} e^{-i x i / \lambda_{T}},
$$

where $\kappa$ is some other appropriate constant containing parameters not of importance here. We now apply (15) with $f(t)=\exp \left(-t^{5 / 3}\right)$, and it can be seen readily that (19) becomes the angular spectrum,

$$
\langle I(Q)\rangle=\kappa e^{-(1 / 2) Q^{2} r^{2}}\left(1+2 \sum_{m=1}^{\infty} e^{-|m T|^{5 / 3}} \cos (m Q T)\right)
$$

where $Q \equiv x / \lambda_{T}=\left(L / \lambda_{T}\right) \tan \theta$ is the normalized angle tangent of the direction of reception. Equation (19) can, alternatively, be considered as the Fourier transform of $\exp \left(-t^{5 / 3}\right)$, and (20) expresses it as a sum of analytically determined terms. Figure 1 exhibits a curve of a normalized $\langle I(Q)\rangle /\langle I(0)\rangle$ vs $Q$ calculated from (20) using $\sigma=T$, and steadily decreasing values of $T$ until convergence of the answer has been reached.

Another class of problems involves a series of integrais dubbed "diffusion integrals" by Hoc, Besieris, and Sockell ${ }^{35}$ and defined by

$$
D f_{N}(x)=\frac{1}{2 \pi} \int_{-\infty}^{\infty} d t e^{-t^{N} / N} e^{-i t x}
$$

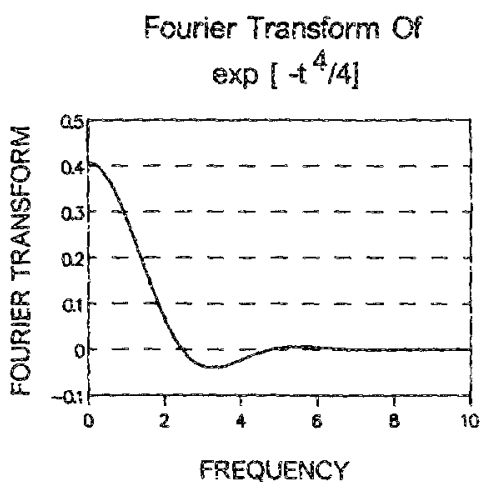

FIG. 2. Logarithm of fractional error in diffusion integral $D f_{2}(x)$.
Errors in F.T. of

$\exp \left[+\left(t^{2}\right) / 2\right]$

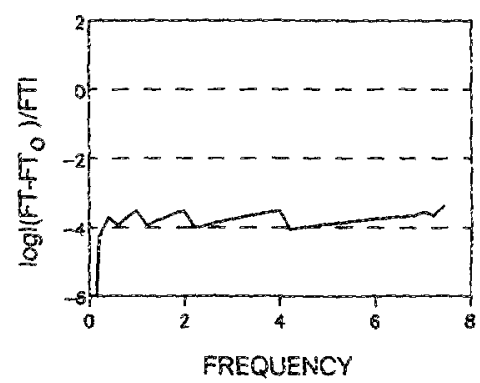

FIG. 3. Diffusion integra! $D f_{4}(x)$ vs $x$

where $N=2,4,6, \ldots$. These integrals occur in the phasespace analysis of pulsed signals in homogeneous, time-invariant, dissipative media. ${ }^{36}$ Application of (15) to $\exp \left(-t^{N} / N\right)$ yields (we will set $\sigma=T$ ),

$D f_{N}(x)=\frac{T}{2 \pi} e^{-\sigma^{2} x^{2} / 2}\left(1+2 \sum_{m=1}^{\infty} e^{-(m T)^{N} / N} \cos (m x T)\right)$.

The exact solution of (21) for $N=2$ is $D f_{2}(x)=(\pi /$ 2) ${ }^{1 / 2} \exp \left(-x^{2} / 2\right)$, which can be used as a check on the numerical evaluation of (22). Figure 2 shows a logarithmic plot of the fractional error for $0<x<7.5$ employed in numerical evaluation of (22) on a $\mathrm{PC}$ with an algorithm that decreases interval $T$ by a factor of 2 until the sum is constant to $0.1 \%$ at fixed $x$. Figures 3 and 4 show plots of $D f_{4}(x)$ and $D f_{6}(x)$, respectiveiy, versus $x$, as calculated with a similar (but higher accuracy) algorithm from (21). Using an asymptotic series ${ }^{36}$ that is accurate for large values of $x$, we have evaluated a logarithmic fractional error for both of these functions in Figs. 5 and 6 . As all of these calculations are done for 30 to 50 values of $x$ on an IBM-PS/2 Model 50 with 80287 co-processor in a compiled BASIC program that takes only a few minutes to execute, the computational aspects may be considered to be fairly trivial once the expansion (15) has been employed in these integrals.

The functions expanded through (15) in this section are not strictly band limited. However, they are all exponentially decaying with $x$ at powers close to or higher than two, and as such have effective bandwidths defined by noise level (accuracy). The above examples bear out that a sufficient decrease in the sampling interval will yield any desired accura-

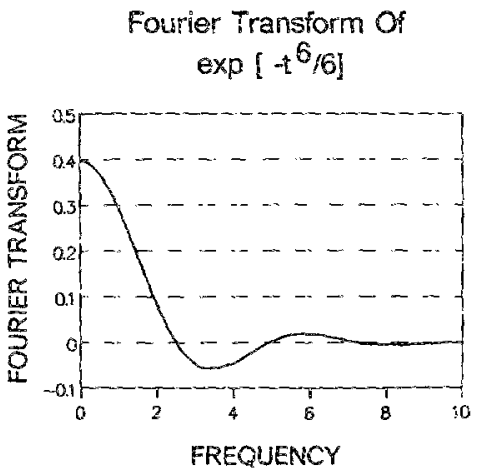

FIG, 4. Difusion integral $D f_{6}(x)$ vs $x$. 


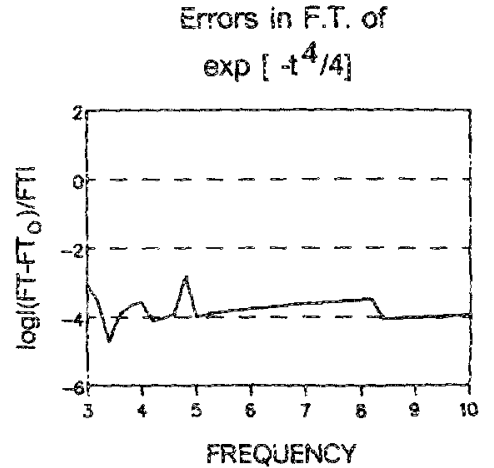

cy. However, as we have stated above, in other practical cases a compromise between desired accuracy and computational cost may be necessary. This is the case especially where higher-dimensional functions are concerned, e.g., as in the expansion of four-dimensional phase-space functions. ${ }^{4,11}$ Nevertheless, the ability to do certain calculations at a relatively bearable cost, even at the sacrifice of some desired accuracy, often may be preferable to any aiternative (such as doing only a small fraction of the calculation at desired accuracy and high cost). For such applications, (15) represents a significantly useful aiternative.

Note added in proof: A reviewer has kindly brought to my attention a recent application in which the Gabor expansion is confined to the $n$ summation. ${ }^{37}$ This is only one of the other possibilities offered by the full bi-orthogonal Gabor expansion itself.

'M. Born and E. Wolf Principles of Optics, 3rd ed. (Pergamon, Oxford, (965).

'B. J. Uscinski, The Elements of Wave Propagation in Random Media (McGraw-Hill, New York, 1977).

${ }^{3}$ A. ishimarn, Wove Propagation and Scattering in Random Media (Acatdemic, New York, 1978).

${ }^{4}$ D. A. de Wolf and J.K. Pack, J. Opt. Soc. Am. A 3, 532 (1986).

${ }^{5}$ A. P. Kachalov and M. M. Popov, Sov. Phys. Dokl. 26, 604 (1981).

${ }^{6}$ V. Cervený, M. M. Popov, and I. Pšenčik, Geophys. J. R. Astron. Soc. 70, 109 (1982).

7M. M. Popov, Wave Motion 4, 85 (1982).

"E. B. Felser, I. Opt. Soc. Am. A 3,486 (1986).

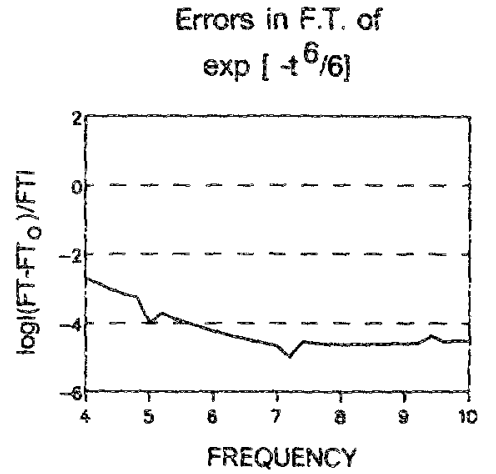

FIG. 6. Logarithm of fractional error in $D f_{6}(x)$.

${ }^{9}$ L. B. Felsen, Geophys. J. R. Astron. Soc. 79, 153 (1984).

1op. G. Mantica, I. Montrosset, R. Tascone, and R. Zich, J. Opt. Soc. Am. A. 3,497 (1986).

13.-K. Pack and D. A. de Wolf, J. Opt. Soc. Am. A 3, 1766 (1986).

${ }^{12}$ G. Müller, Geophys. J. R. Astron. Soc. 79, 153 (1984).

13. Klimeš, Geophys. J. R. Astron. Soc. 79, 105 (1984).

${ }^{14}$ R. Madariaga, Gecphys. J. R. Astron. \$oc. 79, 589 (1984).

${ }^{15}$ E. J. Heiler, J. Chem. Phys 62, 1544 (1975).

16M. J. Davis and E. J. Heller, J. Chem. Phys. 71, 3383 (1979).

"M. J. Davis and E. J. Heller, J. Chem. Phys. 75, 3917 (1981).

${ }^{2} \mathrm{M}$. J. Davis and E. J. Heller, J. Chern. Phys. 80, 5036 (1984).

${ }^{14}$ S.-Y. Lee and E. I. Heller, J. Chem. Phys. 76, 3035 (1982).

${ }^{211}$ G. Drolshagen and E. J. Heller, J. Chem. Phys. 79, 2072 (1983).

${ }^{2}$ R. T. Skodje, Chem. Phys. Lett. 109, 227 (1984).

${ }^{22}$ R. T. Skodje and D. G. Truhlar, J. Chem. Phys. 90, 3123 (1984).

${ }^{2.1}$ S.-I. Sawada, R. Heather, B. Jackson, and H. Metiu, J. Chem. Phys. 83, 3009 (1985).

${ }^{24}$ B. Jackson and H. Metiu, J. Chem. Phys. 83, 1952 (1985).

${ }^{25}$ M. J. Bastiaans, Optik 57,95 (1980).

${ }^{26}$ D. Gabor, J. IEE 93,429 (1946).

${ }^{27}$ A. J. E. M. Janssen, J. Mater. Anval. Appl. 83, 377 (1981).

${ }^{24}$ P. D. Einziger, S. Raz, and M. Shapira, J. Opt. Soc. Am. A 3, 508 (1986).

${ }^{29} \mathrm{H}$. S. Carslaw, An Introduction to the Theory of Fourier's Series and Integrals, 3rd ed. (Dover, New York, 1950).

${ }^{30}$ R. W. Lee and J. C. Harp, Proc. IEEE 57, 375 (1969).

${ }^{31}$ I. G. Yakushkin, Izv. Vyssh. Uchebr. Zaved. Radiofiz. 17, 1350 (1981).

${ }^{32}$ S. I. Belousov and I. G. Yakushkin, Izv. Vyssh. Uchebn. Zaved Radionz. $23,945(1981)$.

${ }^{13}$ E. Jakeman and J. G. McWhirter, J. Phys. A 10, 1599 (1977).

${ }^{34}$ R. L. Fante, Proc. IEEE 63, 1669 (1975).

${ }^{35}$ N. D. Hoc, I. M. Besieris, and M. E. Sockell, IEEE Trans. Antennas Propagat. AP-33, 1237 (1985).

${ }^{36}$ N. D. Hoc, M.Sc. Thesis, VPI\&SU, Blacksburg, VA, 1983.

${ }^{37} \mathrm{~J}$. Maciel and L. B. Felsen, IEEE Trans. Aniemas Propagat. AP.37 (1989). 